\title{
Improved Iterative Parallel Interference Cancellation Receiver for DS-CDMA 3G Systems
}

\author{
Simone Morosi, Enrico Del Re, Romano Fantacci, Andrea Bernacchioni \\ Electronics and Telecommunication Department \\ University of Florence \\ Via di S. Marta, 3, Firenze, 50139, Italy \\ Email: \{morosi, delre, fantacci, bernacchioni\}@lenst.det.unifi.it
}

\begin{abstract}
This paper presents a Turbo Multiuser Detector for Turbo-Coded DS-CDMA systems, based on the utilization of a PIC and a bank of turbo decoders, in which the PIC performs interference cancellation after each constituent decoder of the turbo decoding scheme. Moreover, we propose a new enhanced estimator of the signal-to-noise-plus-interference-ratio used in the decision device and in the MAP decoding algorithm. By means of computer simulations, we will show that the proposed receiver ouperforms other similar schemes for medium to very high system loads, in AWGN and multipath fading channel.
\end{abstract}

\section{INTRODUCTION}

One of the main shortages of the Code Division Multiple Access (CDMA) communication systems can be identified in their vulnerability to Multiple Access Interference (MAI): hence, during the 90's, great attention has been devoted to Multiuser Detection strategies that constitute the natural answer to this problem; the receivers based on this technique aim to exploit the intereference as an additional information source but, generally, are characterized by a very high complexity. Firstly, multiuser detection researches have been focused on uncoded systems; however, practical CDMA communications rely on the utilization of error control coding and interleaving so that, recently, more and more attention has been addressed to coded systems. Optimal joint decoding/detection is an excellent solution to this problem, as shown in [1]. However, this scheme results in a prohibitive computational complexity for actual implementation. In contrast, suboptimal solutions, wich separate the operations of symbol detection and channel decoding, appears more attractive for practical applications.

Particularly, the successful proposal of Turbo codes [2] naturally leads to investigate the feasibility of iterative (Turbo) processing techniques in the design of multiuser receivers. In the iterative multiuser detection, extrinsic information is determined in each detection and decoding stage and used as a priori information for the next iteration. This procedure is adopted at each iteration as in Turbo codes: this detection philosophy is defined as Turbo MUD and the advantages due to its introduction are remarkable, also for heavily loaded systems. In the last years many iterative receivers that can achieve nearoptimal performance [3], [4] have been investigated, but with the drawback of the complexity, that is still exponential in the number of users. This is the reason of the attention addressed to Interference Cancellation schemes [5], [6], [7], [8].

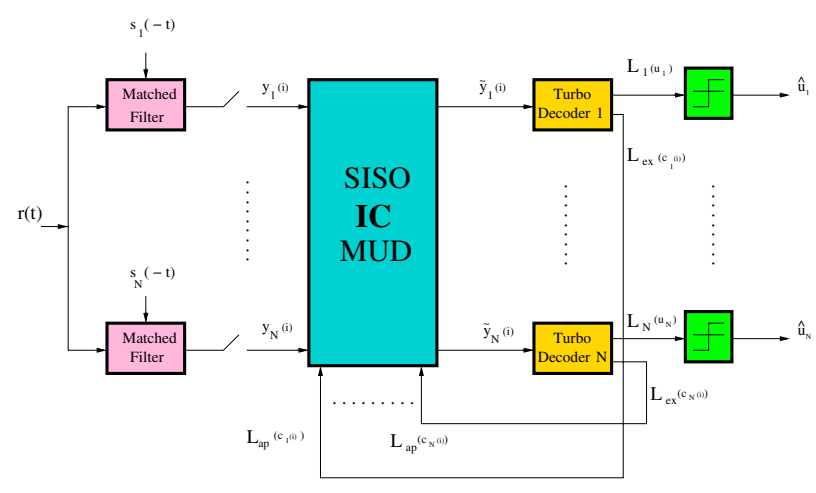

Fig. 1. The general iterative IC receiver

In this paper, we propose a new iterative multiuser detector based on the utilization of a Parallel Interference Cancellation (PIC) and a bank of Turbo decoders. In the proposed structure the PIC detector is broken up so that it is possible to perform Interference Cancellation after each constituent convolutional decoder. Due to the tight relationship between the proposed receiver and the MAP decoders, we defined it as MAP decoder aided PIC (MPIC): this solution aims to profit by IC introduction from the first iterations. Moreover, in the paper the variance of the noise-plus-(residual-)interference is determined by a new algorithm: particularly, only the most reliable symbols are used in variance determination, neglecting all the others. This solution affords performance improvement for all the considered systems.

\section{SySTEM MODEL}

We consider an up-link DS-CDMA communication system with $N$ synchronous turbo-coded users. Timing, amplitudes, carrier phases and spreading sequences of all the users are assumed to be perfectly known at the receiving end in the base station. Each user encodes blocks of information bits $u_{k}(i)$ with a Parallel Concatenated Convolutional Code (PCCC) and transmits the resulting codewords composed of $M$ coded bits over a common AWGN channel with BPSK modulation. The equivalent baseband received signal can be written as

$$
r(t)=\sum_{k=1}^{N} \sqrt{E_{b_{k}}} \sum_{i=0}^{M-1} c_{k}(i) p\left(t-i T_{b}\right) s_{k}\left(t-i T_{b}\right)+n(t)
$$


where:

- $T_{b}$ is the bit interval;

- $E_{b_{k}}$ is the $k$ th user received energy;

- $c_{k}(i) \in\{+1,-1\}$ is the bit transmitted by $k$ user during the $i$ th bit period;

- $p(t)$ is the unit-power rectangular pulse shape with duration $T_{b}$;

- $s_{k}(t)$ is the $k$ th user unit-power spreading sequence;

- $n(t)$ is an Additive White Gaussian Noise (AWGN) process with double sided spectrum density $\sigma^{2}=N_{o} / 2$ [W/hz].

In the receiver a bank of matched filter is used for despreading. Without loss of generality, we can assume that the first bitinterval is observed. As a result, the output of the $k$ th matched filter is given by

$y_{k}=\frac{1}{T_{b}} \int_{0}^{T_{b}} r(t) s_{k}(t) d t=\sqrt{E_{b_{k}}} c_{k}+\sum_{\substack{j=1 \\ j \neq k}}^{N} \sqrt{E_{b_{j}}} c_{j} \rho_{j k}+n_{k}$

where $\rho_{j k}$ is the normalized crosscorrelation coefficient between users $j$ and $k$ and $n_{k}$ is the noise Gaussian sample of user $k$ with distribution $N\left(0, \sigma^{2}\right)$. The second term in eq.(2) represent the MAI, that has to be cancelled.

\section{A New IC Iterative Receiver}

An iterative cancellator consists of an Interference Cancellation (IC) based Multi-User Detector (MUD) followed by $N$ single-user turbo decoders. Each constituent block iteratively provides soft informations to the others, as shown in Fig 1.

In the first multiuser detection iteration, the a priori information of coded bits is not available, i.e. $L_{a p}\left(c_{k}(i)\right)=0$, $\mathrm{k}=1,2, \ldots, \mathrm{N}, \mathrm{i}=0,1, \ldots \mathrm{M}-1$. The IC stage delivers interferencecancelled soft outputs $\tilde{y}_{k}(i)$ to the input of the turbo decoders. After a fixed number of turbo decoder iterations, the extrinsic information of coded bits at the output of turbo decoders are fed back to the input of the IC detector as the a priori information for the next receiver iteration.

The considered turbo codes are composed of two Recursive Systematic Convolutional (RSC) codes linked by an interleaver and a MAP based algorithm is used for iterative decoding [9]. Since the IC receiver requires soft information about reliability of both the systematic and the parity bits, the decoding algorithm is properly modified to produce also extrinsic information about the latter [10]. At each new iteration, the iterative structure permits the multiuser receiver to have a more reliable a priori information and the decoders to operate on soft inputs, in which a greater amount of interference has been cancelled.

\section{A. The Conventional Iterative PIC Receiver}

In the conventional iterative Parallel Interference Cancellation (PIC) receiver [11], at each IC stage, MAI is to be removed simultaneously from each user. Therefore, at the $m$ th receiver iteration, the PIC soft output, i.e., the turbo decoders input, can be expressed as

$$
\begin{aligned}
\tilde{y}_{k}^{(m)} & =y_{k}-\sum_{\substack{j=1 \\
j \neq k}}^{N} \sqrt{E_{b_{j}}} \rho_{k j} \hat{c}_{j}^{(m)} \\
& =\sqrt{E_{b_{k}}} c_{k}+\sum_{\substack{j=1 \\
j \neq k}}^{N} \sqrt{E_{b_{j}}} \rho_{k j}\left(c_{j}-\hat{c}_{j}^{(m)}\right)+n_{k}
\end{aligned}
$$

where $\hat{c}_{j}^{(m)}$ is the estimate of bit $c_{j}$ at iteration $m$. Note that the second summation represents the residual MAI after cancellation.

The data estimates $\hat{c}_{k}^{(m)}$ have been chosen in [11] as the expectation of coded bits, that is

$$
\hat{c}_{k}^{(m)}=\mathrm{E}\left\{c_{k}^{(m)}\right\}=\tanh \left[\frac{1}{2} \mathrm{~L}_{a p}^{(m)}\left(c_{k}\right)\right],
$$

in order to take advantage on the turbo decoders output. The term $\mathrm{L}_{a p}^{(m)}\left(c_{k}\right)$ is the a priori Log-Likelihood Ratio of bit $c_{k}$ at the $m$ th iteration, defined as

$$
\mathrm{L}_{a p}^{(m)}\left(c_{k}\right) \triangleq \log \frac{P^{(m)}\left(c_{k}=+1\right)}{P^{(m)}\left(c_{k}=-1\right)} .
$$

In the first receiver iteration no a priori information is available from the decoder output: hence, for the initializing condition, it is assumed $\mathrm{L}_{a p}^{(0)}\left(c_{k}\right)=0, \mathrm{k}=1,2, \ldots \mathrm{N}$. Instead, in the successive iterations the extrinsic information coming from the decoders can be used, leading to $\mathrm{L}_{a p}^{(m)}\left(c_{k}\right)=\mathrm{L}_{e x}^{(m-1)}\left(c_{k}\right)$.

\section{B. The Proposed Iterative MPIC Receiver}

As it is known, as the number of decoding iterations increases, the coding gain offered by a turbo decoder becomes larger. However, the performance improvement obtained by turbo codes is remarkable in the first iterations, and more and more negligible in the successive ones. This remark suggests to concentrate the significant part of interferencecancellation in the first iterations: for this reason many IC based iterative receiver with a first linear stage have been proposed [7], [8], [12]. Nevertheless, a linear MUD has the drawback of an extremely high computational complexity.

In this paper we present an iterative PIC receiver, wich exploits most interference-cancellation in the first receiver iterations: a conventional iterative receiver tries to cancel the MAI from all the parity and the systematic bits only once at the end of each decoding iteration. Note that in turbo decoding iteration, the first MAP decoder provides extrinsic informations about all the systematic and half of the parity bits while the second decoder gives the extrinsic information about the interleaved systematic bits and the second half of the parity bits; as a consequence, in the proposed receiver the interference-cancellation is performed after each decoding iteration and the systematic bits are cancelled twice. Because of the tight connection between the proposed receiver and the MAP decoders, we have named it as MAP decoders aided PIC (MPIC). The structure of this receiver is shown in Fig. 2.

Assuming that $R_{c}$ is the turbo encoder code rate and that only the parity bits are punctured at the transmitter end, the 


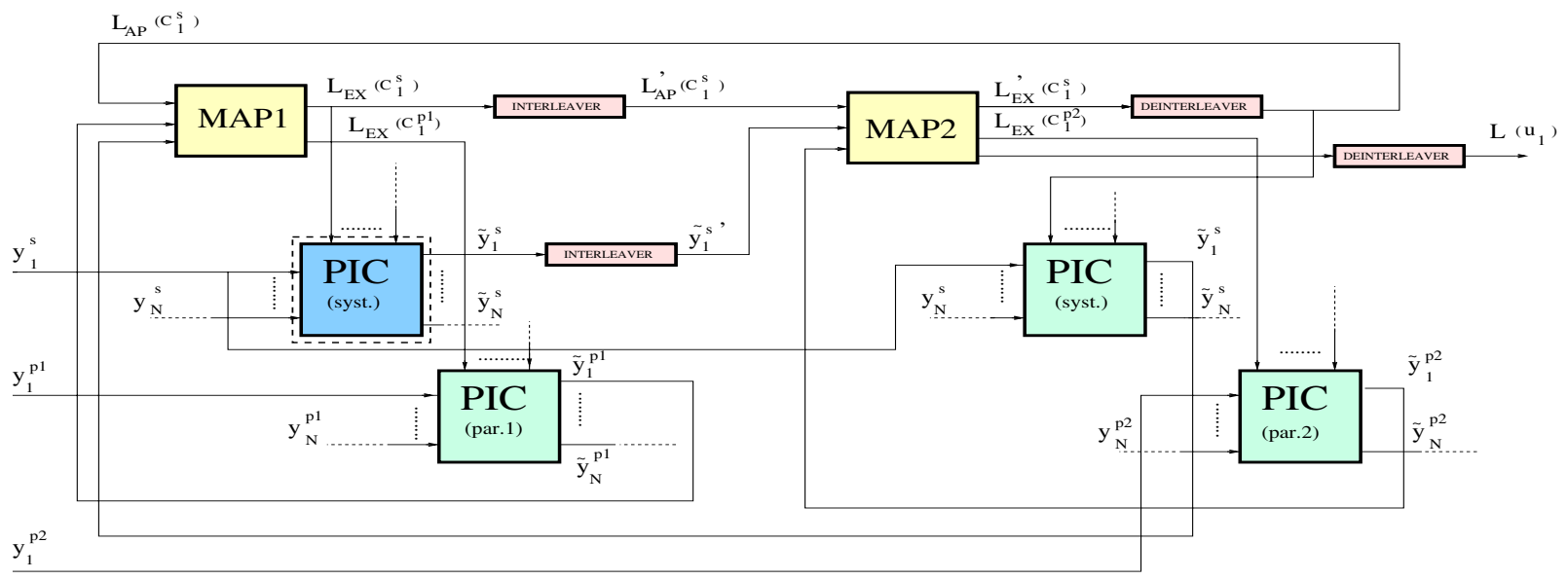

Fig. 2. Block diagram of the proposed iterative MUD.

computational complexity needed by the MPIC to perform interference cancellation at each iteration is $\left(1+R_{c}\right)$ greater than the conventional PIC. However it will be shown by simulation that the proposed iterative receiver performance is better than the conventional one even with equal complexity. This goal has been reached by removing in the last iterations so many cancellation stages as the ones added in the first iterations.

\section{An Improved Non-Linear MMSE Estimator}

The tentative decision device (4), used to estimate the data bits and reconstruct and cancel MAI, exploits only extrinsic informations from the decoders but not channel outputs; hence, only a fraction of available information sources is used in the decision. An improved data estimator can be obtained by exploiting jointly decoders and channel outputs, as in [6], where a ML estimator is used, producing tentative hard decisions [14]. Unlike [6], we derive a non linear MMSE estimator, that can also provide the reliability value of each estimated bit. The decision $\hat{c}_{k}^{(m)}$, for $k$ th user at the $m$ th receiver iteration, is taken as the expectation of $c_{k}$, given the channel output and the a priori probability, i.e.,

$$
\hat{c}_{k}^{(m)}=\mathrm{E}\left\{c_{k} \mid y_{k}, \mathrm{P}\left(c_{k}\right)\right\}=\sum_{c_{k} \in\{+1,-1\}} c_{k} \mathrm{P}\left(c_{k} \mid \tilde{y}_{k}, \mathrm{P}\left(c_{k}\right)\right)
$$

Making the assumption that the interference can be considered as a Gaussian process, we obtain

$$
\hat{c}_{k}^{(m)}=\tanh \left[\frac{1}{2}\left(2 \frac{\sqrt{E_{b_{k}}} y_{k}}{\sigma_{k}^{2}}+\mathrm{L}_{a p}^{(m)}\left(c_{k}\right)\right)\right]
$$

where $\sigma_{k}^{2}$ is the termal noise-plus-interference variance, given by $\sigma_{k}^{2}=\sigma^{2}+\sigma_{k, M A I}^{2}$. As it is shown in [13], combination of channel output and extrinsic information in decision statistic yields a biased residual interference term wich tends to cancel the useful signal. However, computer simulations confirm that better performance is achieved by using all the information sources and that mitigation of the bias effect is obtained after few iterations.

\section{Variance Estimation}

The tentative decision device needs to know the noise-plus(residual)interference variance, such as the channel reliability value used in the MAP decoding [2]; hence, an appropriate estimation method has to be used. In [15] and [5] a simple algorithm based on the data samples has been proposed: particularly, therein the variance is calculated as

$$
\hat{\sigma}_{k, m}^{2}=\frac{1}{M} \sum_{i=0}^{M-1}\left(\tilde{y}_{k}^{(m)}(i)\right)^{2}-{\overline{\tilde{y}_{k}^{(m)}}}^{2},
$$

where the mean $\overline{\tilde{y}_{k}^{(m)}}$ has been either derived by the samples [15] or assumed equal to the square root of the received energy $\sqrt{E_{b_{k}}}$ [5]. As it will be confirmed by simulation results, this algorithm leads to underestimate the variance in the cases of low signal-to-interference-plus-noise-ratio (SINR). The motivaton of this behaviour is the following. Assuming that the (residual-)interference is Gaussian, the conditional probability density functions (pdf) of the variable $\tilde{y}_{k}^{(m)}(i)$, i.e., $p\left(\tilde{y}_{k}^{(m)}(i) \mid c_{k}= \pm 1\right)$, are also Gaussian. As a consequence, the unconditional pdf of $\tilde{y}_{k}^{(m)}(i)$ is given by

$$
\begin{aligned}
p\left(\tilde{y}_{k}^{(m)}\right) & =P\left(c_{k}=+1\right) p\left(\tilde{y}_{k}^{(m)} \mid c_{k}=+1\right) \\
& +P\left(c_{k}=-1\right) p\left(\tilde{y}_{k}^{(m)} \mid c_{k}=-1\right) \\
& =\frac{1}{2}\left[p\left(\tilde{y}_{k}^{(m)} \mid c_{k}=+1\right)+p\left(\tilde{y}_{k}^{(m)} \mid c_{k}=-1\right)\right],
\end{aligned}
$$

where the last equality follows from the assumption that the transmitted bits are indipendent identically distributed (i.i.d.). As it can be seen from Fig.3, the values $\tilde{y}_{k}^{(m)}(i)$, satisfing the inequality $\left|\tilde{y}_{k}^{(m)}(i)\right|<\sqrt{E_{b_{k}}}$, are ambiguous, in the sense that they belong to the region of maximum superposition of the conditional pdfs. For this reason we have chosen to evaluate the variance only by the values satisfing $\left|\tilde{y}_{k}^{(m)}(i)\right| \geq \sqrt{E_{b_{k}}}$; these values has been defined as $\tilde{y}_{k, u p}^{(m)}(i)$. This original variance definition can be used to improve the variance estimation 


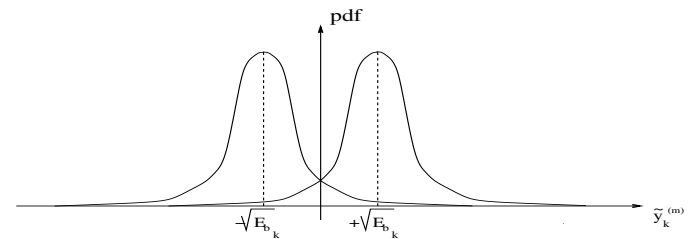

Fig. 3. Decision variable probability density function.

through the formula:

$$
\hat{\sigma}_{k, m}^{2}=\frac{1}{M_{1}} \sum_{i=0}^{M_{1}-1}\left(\left|\tilde{y}_{k, u p}^{(m)}(i)\right|-\sqrt{E_{b_{k}}}\right)^{2}
$$

where $M_{1} \leq M$ indicates the number of such values in a codeword.

\section{ITERATIVE MPIC FOR MUlTiPATH SLOWLY FADING CHANNELS}

Wireless CDMA systems usually have to cope multipath fading environments. The multipath fading channel is often assumed to be wide-sense stationary with uncorrelated scattering [16]. Based on this assumption, the received baseband DS-CDMA signal can be written as

$r(t)=\sum_{k=1}^{N} \sum_{i=0}^{M-1} \sum_{l=1}^{L} \sqrt{E_{b_{k}}} c_{k}(i) \alpha_{k, l}(t) s_{k}\left(t-i T_{b}-\tau_{k, l}\right)+n(t)$

where $L$ is the number of propagation paths and $\alpha_{k, l}(t)$ and $\tau_{k, l}$ are, respectively, the time-varying complex attenuation factor and the relative delay of the $l$ th path of the $k$ th user signal. Besides, we assume that $\mathrm{E}\left\{\sum_{l=1}^{L}\left\|\alpha_{k}, l\right\|^{2}\right\}=1 / T_{b}$, so that $E_{b_{k}}$ is the received averaged energy of $k$ th user, and that channel fading is so slow that $\alpha_{k, l}(t)$ does not change in a chip interval and thus can be denoted as $\alpha_{k, l}(h)$, where $h$ refers to chip position in the codeword. The chip matched filter output for the $l$ th path of the $k$ th user is then given by

$$
y_{k, l}(h)=\frac{1}{T_{c}} \int_{(h-1) T_{c}+\tau_{k, l}}^{h T_{c}+\tau_{k, l}} r(t) s_{k}\left(t-\tau_{k, l}-\left\lfloor\frac{h}{G}\right\rfloor T_{b}\right) d t,
$$

where $T_{c}$ is the chip interval, that is tied to the bit interval through the processing gain $G=T_{b} / T_{c}$.

If a Maximal Ratio Combiner (MRC) RAKE receiver is used, the decision statistic can be expressed as

$$
y_{k}(i)=\sum_{l=1}^{L} \sum_{h=i G}^{(i+1) G-1} \alpha_{k, l}^{*}(h) y_{k, l}(h) \quad i=0,1, \ldots, M-1 .
$$

In the $m$ th iteration of the iterative PIC receiver, the $k$ th received signal after MAI subtraction is given by

$r_{k}^{(m)}(t)=r(t)-\sum_{\substack{j=1 \\ j \neq k}}^{N} \sum_{i=0}^{M-1} \sum_{l=1}^{L} \sqrt{E_{b_{j}}} \hat{c}_{j}^{(m)}(i) \alpha_{j, l}(t) s_{j}\left(t-i T_{b}-\tau_{j, l}\right)$

where the bit tentative decision $\hat{c}_{j}^{(m)}(i)$ is given by (7), while the variance estimation formula has to be modified
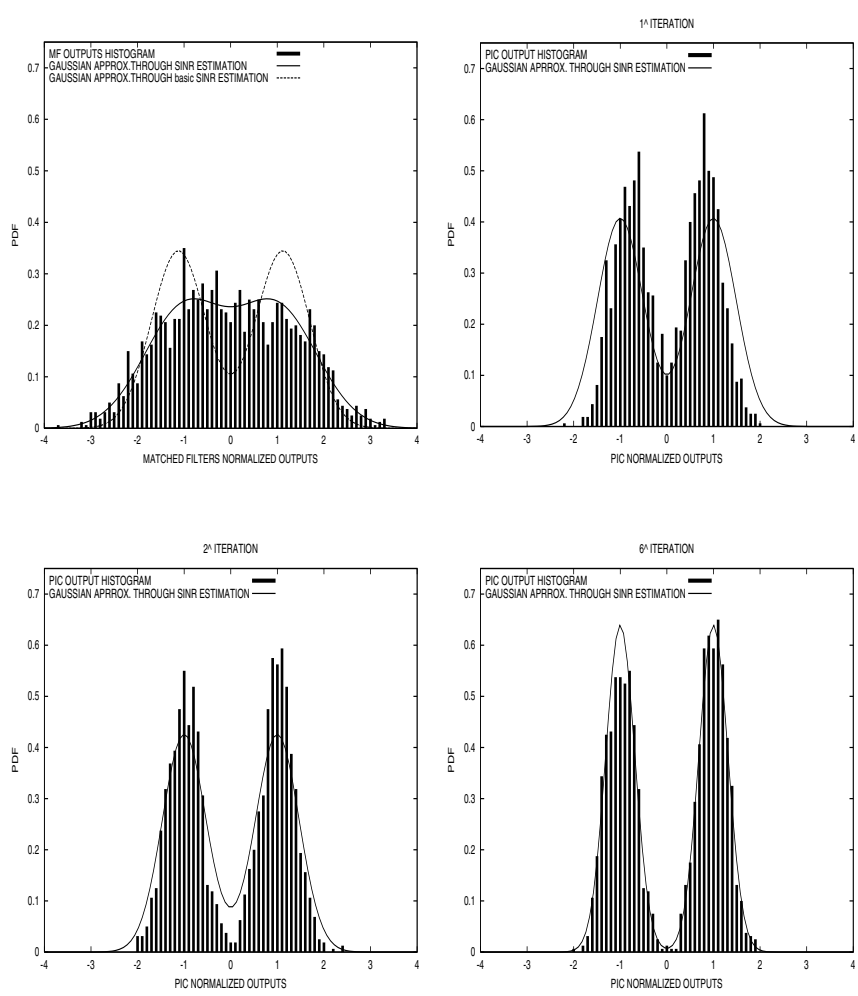

Fig. 4. Histograms and Gaussian approximations of Matched Filters and PIC output for different iterations: $E_{b} / N_{0}=10 \mathrm{~dB}$

to take into account the fading attenuation. The conditional pdfs $p\left(\tilde{y}_{k}^{(m)}(i) \mid c_{k}= \pm 1, \alpha_{k, l}\right)$ are Gaussian and (10) can be rewritten as

$$
\hat{\sigma}_{k, m}^{2}=\frac{1}{M_{1}} \sum_{i=0}^{M_{1}-1}\left(\left|\tilde{y}_{k, u p}^{(m)}(i)\right|-\overline{\alpha_{k, u p}(i)} \sqrt{E_{b_{k}}}\right)^{2}
$$

where $\overline{\alpha_{k}(i)}$ is the bit-averaged fading attenuation after recombination and it is computed as

$$
\overline{\alpha_{k}(i)}=\frac{1}{G} \sum_{h=1}^{G} \sqrt{\sum_{l=1}^{L}\left\|\alpha_{k, l}(i G+h)\right\|^{2}} \text {. }
$$

\section{Simulation Results}

In this section we investigate the effectiveness of the proposed receiver through computer simulations. In order to mitigate the complexity burden due to the implementation of a non-linear decision device, the $\tanh ()$ function has been approximated through an eight-values look-up table. For all simulations we use a rate $R_{c}=1 / 2$ turbo code, composed by two 8 -state RSC codes with generator polynomials $G_{0}=(13)_{8}$ $G_{1}=(15)_{8}$, and the Block Interleaver recommended by the UMTS standard [17]. We compare the proposed iterative MPIC receiver with the conventional PIC and the iterative Partial PIC (PPIC) receiver proposed in [6] and derived from [14]. 
First, we analize the performance in a synchronous AWGN channel. The system has 10 equal-power users with PseudoNoise short codes, processing gain $G=16$ and frame lenght 800. The quantized Log-MAP algorithm is used for the decoding [9]. Fig.4 shows the decision variables histogram at the output of the MF bank and of the PIC receiver after 1, 2, 3 and 6 iterations for a signal-to-noise-ratio $E_{b} / N_{0}=10 \mathrm{~dB}$, compared with the Gaussian distribution with variance calculated through (10). We can remark the interference reduction through iterations and the accuracy of enhanced variance estimation, while a bias is evident just in the first iteration. In Fig. 5 it is shown the performance improvement obtained using the enhanced variance estimator in comparison with the basic one, derived from (8). Fig.6 shows the performance gain achieved by the proposed receiver by increasing the number of iterations. At a BER of $10^{-4}$ and with 16 iterations the loss from single-user bound is about $0.8 \mathrm{~dB}$. A comparison of performance vs iterations between the iterative PIC, MPIC and PPIC is made in Fig.7 at $E_{b} / N_{0}=3 \mathrm{~dB}$. The cancellation weights for PPIC have been found by a trial-and-error searching method, as $p_{0}=0.55, p_{1}=0.65, p_{2}=0.75, p_{3}=0.85, p_{4}=0.92$, $p_{5}=0.97, p_{i \geq 6}=1.0$.

Then, we illustrate the performance of the proposed receiver in a asynchronous 3-paths fading channel with perfect power control: the replica amplitudes are assumed to be Rayleigh distributed, with relative attenuations equal to $0,-1,-9 \mathrm{~dB}$. Processing gain is $G=15$ and spreading is performed by a set of Pseudo-Noise short codes. The frame lenght is 500, a MRC-RAKE receiver is used as paths-combiner and the Max-Log-MAP algorithm is used for decoding. The channel has a normalized frequency Doppler $f_{d} T_{b}=0.0002$ and just 2 receiver iterations are performed. The other transmission parameters are the same of the AWGN case. In Fig. 8 it can be seen that in a system with 20 active users, i.e.with a system load $\beta=N / G$ greater than one $(\beta=1.33)$, the MPIC outperforms the conventional PIC: a gain of $3.5 \mathrm{~dB}$ for the MPIC versus the PIC receiver at a BER of $10^{-3}$ is observed. In this figure it is also shown the great benefit obtained for both the receivers by using a correct estimate of the noiseplus-(residual)interference variance rather than approximating it by the ambient noise, assumed perfectly known, as in [6]. Fig.9 shows that the iterative MPIC receiver can supports heavily loaded DS-CDMA systems. This behaviour can be exalted with the assistance of low crosscorrelations spreading sequences, as shown in Fig.10 where the MPIC receiver reaches single-user performance in a fully loaded DS-CDMA system, with normalized Doppler frequency $f_{d} T_{b}=0.0004$, processing gain 31 and 31 equal-power users using Gold31 codes.

\section{CONCLUSION}

In this paper a new Turbo Multiuser Detector for turbocoded DS-CDMA systems has been presented. In the proposed detector a Parallel Interference Cancellation (PIC) has been divided in order to perform interference cancellation after each MAP constituent decoder. Moreover, an improved algorithm

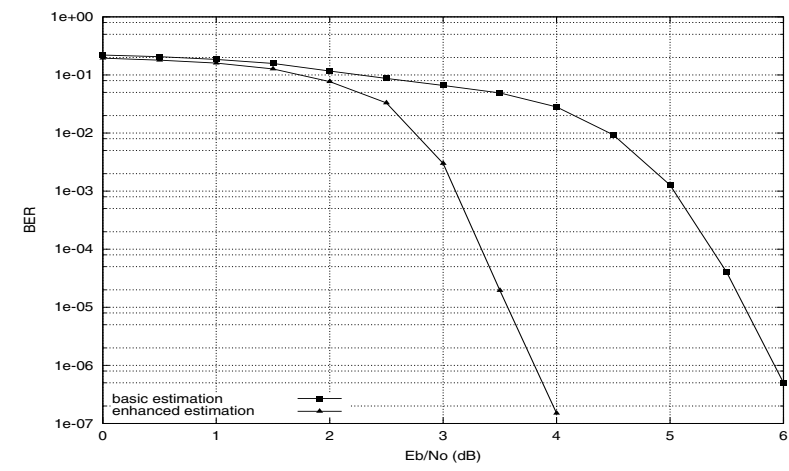

Fig. 5. Performance comparison of iterative PIC receiver with basic and enhanced variance estimator in a synchronous AWGN channel with 10 equalpower users and processing gain 16.

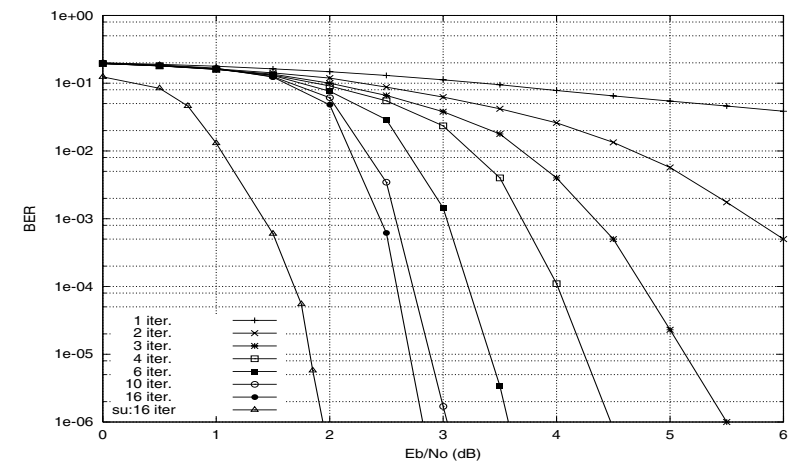

Fig. 6. BER versus $E_{b} / N_{0}$ of iterative MPIC through iterations in an AWGN channel.

to estimate the variance of the noise-plus-residual-interference has been derived. Performance results, evaluated by means of computer simulations for different system loads, in AWGN and multipath fading channel, confirms the excellent behaviour of the proposed receiver. It is shown that it outperforms recently proposed alternative IC based turbo MUD with the same complexity.

\section{ACKNOWLEDGMENT}

The authors would like to thank Eng. Francesco Chiti for his fruitful suggestions and discussions.

\section{REFERENCES}

[1] T. R. Giallorenzi and S. G. Wilson, "Multiuser ML sequence estimator for convolutionally-coded asynchronous DS-CDMA systems," IEEE Transactions on Communications, Vol. 44, pp. 997-1008, August 1996.

[2] C. Berrou, A. Glavieux and P. Thitimajshima, "Near Shannon limit errorcorrecting coding and decoding: Turbo codes," in Proc. 1993 Int. Conf. on Communications (ICC'93), Washington DC, USA, pp. 34, June 2001.

[3] M. C. Reed, C. B. Schlegel, P. D. Alexander J. A. Asenstorfer, "Iterative Multiuser detection for CDMA with FEC: Near Single-User Performance," IEEE Trans. on Commun., Vol. 46, pp. 1693-1699, Dec. 1998.

[4] M. Moher, "An Iterative Multiuser Decoder for Near-Capacity communications," IEEE Trans. on Commun., Vol. 46, pp. 870-880, July 1998.

[5] M. Kobayashi, J. Boutros and G. Caire, "Iterative Soft-SIC Joint decoding and Parameter Estimation," in DSP 2001 7th Int. Workshop on Digital Signal Proc. Techn. for Space Commun., Sesimbre, Portugal, Oct. 2001. 


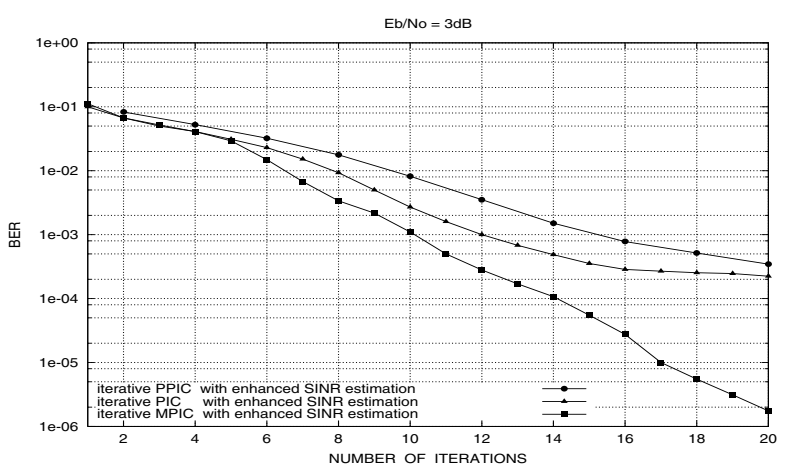

Fig. 7. Performance of different iterative IC receivers against iterations in an AWGN channel.

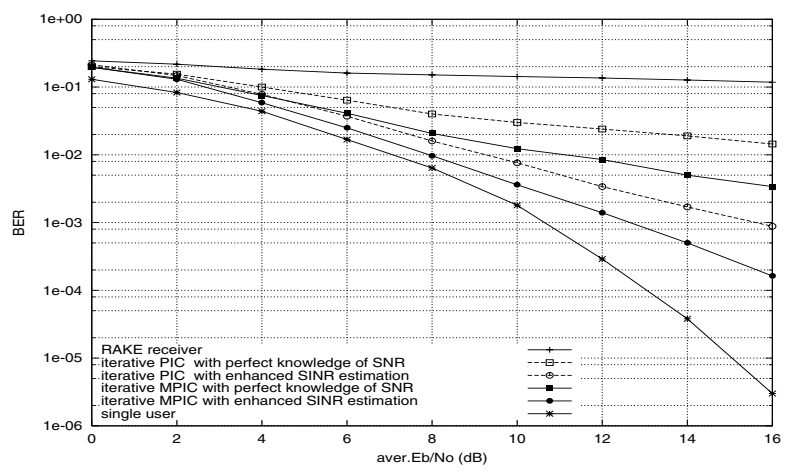

Fig. 8. Performance of iterative IC receivers in a multipath fading channel with 20 equal-power users.

[6] K.-M. Wu and C.-L. Wang, "An Iterative Multiuser receiver Using Partial Parallel Interference Cancellation for Turbo-Coded DS-CDMA Systems," in Proc. of GLOBECOM 2001, S.Antonio, TX, USA, pp. 244-248, November 2001.

[7] Z. Qin and K. C. Teh, "Iterative multiuser detection with Gauss-Seidel soft detector as first stage for asynchronous coded CDMA," Electronics Letters, Vol. 36, No. 23 pp. 1939-1940, November 2000.

[8] J.-M. Hsu and C.-L. Wang, "A Low-Complexity Iterative Multiuser Receiver for Turbo-Coded DS-CDMA Systems," IEEE Journal on Selected Areas in Communications, Vol. 19, pp. 1775-1783, September 2001.

[9] P. Robertson, E. Villebrun and P. Hoeher, "A Comparison of Optimal and Sub-Optimal MAP Decoding Algorithms Operating in the Log Domain," in Proc. Intern. Conf. Comm. (ICC'95), pp. 1009-1013, June 1995.

[10] X. Wang and H. V. Poor, "Iterative (Turbo) Soft Interference Cancellation and Decoding for coded CDMA," IEEE Transactions on Communications, Vol. 47, No.7, pp. 1046-1061, July 1999.

[11] S. Kim and J. H. Lee, "Performance of Iterative Multiuser Detection with a Partial PIC Detector and Serially Concatenated Codes," in Proc. VTC 2001, Vol. 1, pp. 487-491, 2001.

[12] A. Tarable, G. Montorsi and S. Benedetto, "A Linear Front End for Iterative Soft Interference Cancellation and Decoding in Coded CDMA," in Proc. Int. Conf. on Communications (ICC 2001), Helsinki, Finland, pp. 1-5, June 2001.

[13] S. Marinkovic, B. Viucetic and J. Evans, "Improved iterative interference cancellation," in Proc. ISIT 2001, Geneva, Switzerland, pp. 1064-1070, May 2001.

[14] D. Divsalar, M. K. Simon and D. Raphaeli, "Improved Parallel Interference Cancellation for CDMA," IEEE Transactions on Communications, Vol. 46, No.2, pp. 258-268, February 1998.

[15] K. Kettunen, "Iterative Multiuser Receiver/Decoders with Enhanced Variance Estimation," in Proc. IEEE 49th Vehicular Technology Conference, Houston, TX, USA, pp. 2318-2322, May 1999.

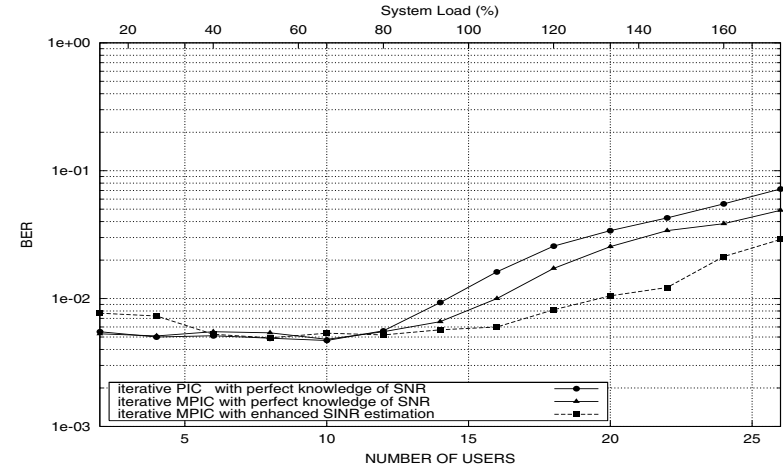

Fig. 9. BER versus System Load for iterative IC receivers in a multipath fading channel.

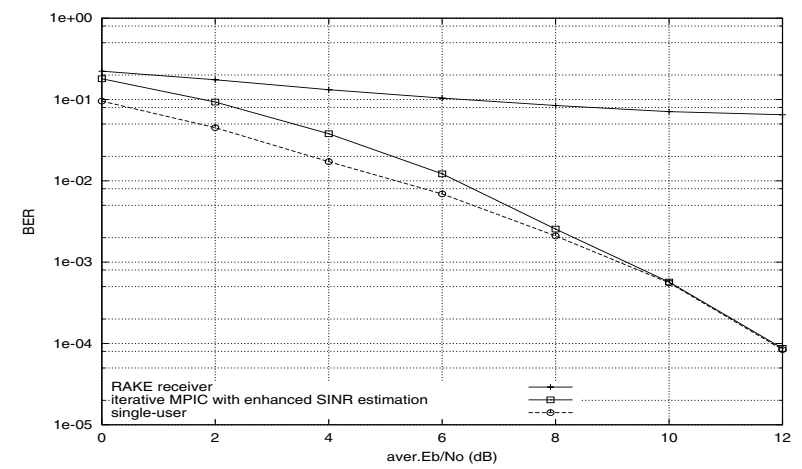

Fig. 10. Performance of iterative MPIC receiver in a multipath fading channel with 31 equal-power users using Gold31 spreading sequences.

[16] J. G. Proakis, Digital Communications, 3rd ed. New York: McGrawHill, 1995.

[17] 3GPP, TR 25.221 multiplexing and channel coding, Tech. Rep., 3GPP, 2001. 